\title{
NOTE ON METRIC DIMENSION
}

\author{
REESE T. PROSSER
}

ABstract. The metric dimension of a compact metric space is defined here as the order of growth of the exponential metric entropy of the space. The metric dimension depends on the metric, but is always bounded below by the topological dimension. Moreover, there is always an equivalent metric in which the metric and topological dimensions agree. This result may be used to define the topological dimension in terms of the metric entropy as the infimum of the metric dimension taken over all metrics.

Kolmogorov's concept of $\epsilon$-entropy, defined for compact metric spaces [3], has proved a useful measure of the "bulk" of such a space. In this note we relate this concept to the Hausdorff and topological dimensions of spaces for which these dimensions are defined. Our result appears as Theorem 2 below.

Let $X$ be a compact metric space, with distance function $\rho$. If $U$ is any subset of $X$, the diameter of $U$ is the finite number

$$
\delta(U)=\sup \{\rho(x, y): x, y \in U\} .
$$

If $\mathcal{u}$ is any family of subsets $U$ of $X$, then the mesh of $\mathcal{u}$ is the number

$$
\mu=\sup \{\delta(U): U \in \mathcal{u}\} .
$$

Every open cover of $X$ admits a finite refinement of arbitrarily small mesh. The size of a finite open cover is the number of sets in the cover; the order is one less than the number of sets in the largest subfamily having nonempty intersection.

Definition. $X$ has topological dimension $\leqq n$ iff for each $\eta>0$ there exists a finite open cover of mesh $<\eta$ and order $\leqq n$. In this case we put

$$
\operatorname{td}(X)=\min \{n: \mathrm{t}-\operatorname{dim} X \leqq n\} .
$$

The equivalence of this definition with Urysohn's original definition of $\operatorname{td}(X)$ is proved in [2, Chapter $\mathrm{V}]$.

Definition. $X$ has Hausdorff dimension $\leqq p$ iff for each $\eta>0$ there exists a finite open cover $\left\{U_{i}\right\}$ of $X$ such that $\sum_{i=1}^{N} \delta\left(U_{i}\right)^{p}<\eta$. In this case we put

$$
\operatorname{hd}(X)=\inf \{p: \operatorname{h}-\operatorname{dim}(X) \leqq p\}
$$

Received by the editors May 23, 1969.

AMS Subject Classifications. Primary 5435, 5470; Secondary 4141.

Key Words and Phrases. Metric dimension, topological dimension, metric entropy. 
The equivalence of this definition with Hausdorff's original definition [1] of hd $(X)$ when $X$ is compact is proved in [2, Chapter VII].

Definition. $X$ has metric dimension $\leqq r$ iff for every $\eta>0$ there exists a finite open cover of size $N$ and mesh $\epsilon$ such that $N \epsilon^{r}<\eta$. In this case we put

$$
\operatorname{md}(X)=\inf \{r: \mathrm{m}-\operatorname{dim} X \leqq r\} .
$$

It is straightforward to verify that this definition of $\mathrm{md}(X)$ is equivalent to the definition of $\operatorname{md}(X)$ as the order of growth of the exponential $\epsilon$-entropy $N(\epsilon, X)=\exp H(\epsilon, X)$ of $X$ :

$$
\operatorname{md}(X)=\inf \left\{r: N(\epsilon, X) \leqq \operatorname{const}(1 / \epsilon)^{r}\right\} .
$$

Lemma 1. For any compact metric space $X$ we have

$$
\operatorname{td}(X) \leqq \operatorname{hd}(X) \leqq \operatorname{md}(X) .
$$

Proof. The first inequality is proved in [2, Chapter VII]. The second stems from the observation that for any open covering of size $N$ and mesh $\epsilon$, we have $\delta\left(U_{i}\right) \leqq \epsilon$, and hence

$$
\sum_{i=1}^{N} \delta\left(U_{i}\right)^{r} \leqq N \epsilon^{r}
$$

Thus if $\mathrm{m}-\operatorname{dim} X \leqq r$, then $\mathrm{h}-\operatorname{dim} X \leqq r$, and so hd $(X) \leqq \operatorname{md}(X)$.

These dimensions all agree on simple spaces, e.g. on all simplicial polytopes in $R^{n}$, but they may differ on spaces of more complicated structure. Thus for example if $X$ is the classical Cantor set in the unit interval $[0,1]$, then we have $\operatorname{td}(X)=0, \operatorname{hd}(X)=\log 2 / \log 3$, $[1],[2]$; while for certain other perfect subsets of $[0,1]$ we have $\operatorname{hd}(X)=0, \operatorname{md}(X)=1,[4]$. It is therefore reassuring to know that $X$ can always be remetrized so that the three definitions agree.

THEOREM 2. For any compact metric space $X$ there exists an equivalent metric such that

$$
\operatorname{td}(X)=\operatorname{hd}(X)=\operatorname{md}(X) .
$$

Proof. The fact that a metric exists such that $\operatorname{td}(X)=\operatorname{hd}(X)$ is proved in $[2$, Chapter VII]. Essentially the same proof will yield a metric such that $\operatorname{td}(X)=\operatorname{md}(X)$, as follows.

If $\operatorname{td}(X)$ is infinite, there is nothing to prove. Otherwise, let $n=\operatorname{td}(X)$, and for $r>n$ consider the class $K_{r}$ of all mappings $f$ $\in C\left(X, I_{2 n+1}\right)$, where $I_{2 n+1}$ is the unit cube in $R^{2 n+1}$, such that $\operatorname{md}(f(X))<r$. Since $f(X)$ is compact, there exists for every integer $k$ an open cover of size $N$ and mesh $\epsilon$ such that $N \epsilon^{r}<1 / k$. If we put 
$K_{k, r}$ the class of all mappings $f$ for which such a cover exists, then

$$
K_{r}=\bigcap_{k=1}^{\infty} K_{k, r}
$$

But $K_{k, r}$ is clearly open in the topology of uniform convergence in $C\left(X, I_{2 n+1}\right)$, so that $K_{r}$ is a $G_{\delta}$-subset of $C\left(X, I_{2 n+1}\right)$.

Now let $K^{*}$ be the class of all mappings $f \in C\left(X, I_{2 n+1}\right)$ such that $f(X)$ is contained in an $n$-dimensional polytope in $I_{2 n+1}$. Then $K^{*}$ is dense in $C\left(X, I_{2 n+1}\right)$ (see $[2$, p. 70$]$ ), and because $r>n$ we have $K^{*} \subset K_{r}$. Hence $K_{r}$ is a dense $G_{\delta}$-subset of $C\left(X, I_{2 n+1}\right)$.

Now let $H$ be the class of homeomorphisms in $C\left(X, I_{2 n+1}\right)$. Then $H$ is also a dense $G_{\delta}$-subset of $C\left(X, I_{2 n+1}\right)$ (see $\left.[2, \mathrm{p} .56]\right)$, and hence the set

$$
H^{*}=H \cap \bigcap_{i=1}^{\infty} K_{n+1, i}
$$

is also a dense $G_{\delta}$-subset of $C\left(X, I_{2 n+1}\right)$ by the Baire category theorem. If $h \in H^{*}$, then evidently $h: X \rightarrow I_{2 n+1}, h$ is a homeomorphism, and $\operatorname{md}(h(X)) \leqq n$. The Euclidean metric on $h(X)$ provides the desired remetrization for $X$.

These results suggest the following alternative definition of the topological dimension of a metrizable space. Let $X$ be metrizable, and write $\mathrm{t}-\operatorname{dim} X \leqq n$ iff there exists a metric for $X$ (compatible with the topology of $X$ ) such that $\mathrm{m}-\operatorname{dim} X \leqq n$. With this definition it is clear that $\operatorname{td}(X)$ is a topological invariant. Moreover, it is relatively easy to derive most of the standard operational properties of $\operatorname{td}(X)$. It is relatively hard, however, to prove that $\operatorname{td}(X)$ is an integer!

\section{BIBLIOGRAPHY}

1. F. Hausdorff, Dimension und ausseres Mass, Math. Ann. 79 (1919), 157-179.

2. W. Hurewicz and H. Wallman, Dimension theory, Princeton Math. Series, vol. 4, Princeton Univ. Press, Princeton, N.J., 1941. MR 3, 312.

3. A. N. Kolmogorov, On the Shannon theory of information transmission, IRE Trans. Information Theory (1956), 102-108.

4. A. C. Vosburg, On the relationship between Hausdorff dimension and metric dimension, Pacific J. Math. 23 (1967), 183-187. MR 36 \#865.

Dartmouth College, Hanover, New Hampshire 03755 